\title{
Imaginary vectors in the dual canonical basis of $U_{q}(\mathfrak{n})$
}

\author{
Bernard LECLERC
}

\begin{abstract}
Let $\mathfrak{n}$ be the maximal nilpotent subalgebra of a simple complex Lie algebra $\mathfrak{g}$. We introduce the notion of imaginary vector in the dual canonical basis of $U_{q}(\mathfrak{n})$, and we give examples of such vectors for types $A_{n}(n \geqslant 5), B_{n}(n \geqslant 3), C_{n}(n \geqslant 3), D_{n}(n \geqslant 4)$, and all exceptional types. This disproves a conjecture of Berenstein and Zelevinsky about $q$-commuting products of vectors of the dual canonical basis. It also shows the existence of finite-dimensional irreducible representations of quantum affine algebras whose tensor square is not irreducible.
\end{abstract}

\section{Introduction}

Let $\mathfrak{g}$ be a simple complex Lie algebra, $U_{q}(\mathfrak{g})$ the corresponding quantized enveloping algebra, $\mathfrak{n}$ a maximal nilpotent subalgebra of $\mathfrak{g}$, and $U_{q}(\mathfrak{n})$ the corresponding subalgebra of $U_{q}(\mathfrak{g})$. Let $\mathbf{B}$ be the canonical basis of $U_{q}(\mathfrak{n})$ [L1, K1], and let $\mathbf{B}^{*}$ be the basis dual to $\mathbf{B}$ for the natural scalar product on $U_{q}(\mathfrak{n})$. In this note, we discuss the multiplicative structure of $\mathbf{B}^{*}$. We write $q^{\mathbb{Z}} \mathbf{B}^{*}=\left\{q^{m} b \mid m \in \mathbb{Z}, b \in \mathbf{B}^{*}\right\}$. For $u, v$ in $U_{q}(\mathfrak{n})$ we also use the short-hand notation $u \cong v$ to mean that there exists $m \in \mathbb{Z}$ such that $u=q^{m} v$.

Definition 1 We say that $b \in \mathbf{B}^{*}$ is real if $b^{2} \in q^{\mathbb{Z}} \mathbf{B}^{*}$. Otherwise, we say that $b$ is imaginary.

In [BZ] it was conjectured that, given $b_{1}, b_{2} \in \mathbf{B}^{*}$, the product $b_{1} b_{2}$ is in $q^{\mathbb{Z}} \mathbf{B}^{*}$ if and only if $b_{1}$ and $b_{2} q$-commute with each other, i.e. $b_{2} b_{1} \cong b_{1} b_{2}$. In particular, taking $b_{1}=b_{2}$, this conjecture would imply that all vectors of $\mathbf{B}^{*}$ are real. In Section 2 below, we give examples of imaginary vectors in $U_{q}(\mathfrak{n})$ for $\mathfrak{g}$ of type $A_{5}, B_{3}, C_{3}, D_{4}$ and $G_{2}$. Using the natural embeddings $U_{q}\left(A_{5}\right) \subset U_{q}\left(A_{n}\right)(n \geqslant 6), U_{q}\left(B_{3}\right) \subset U_{q}\left(B_{n}\right)(n \geqslant 4), U_{q}\left(C_{3}\right) \subset U_{q}\left(C_{n}\right)(n \geqslant 4), U_{q}\left(D_{4}\right) \subset$ $U_{q}\left(D_{n}\right)(n \geqslant 5), U_{q}\left(D_{4}\right) \subset U_{q}\left(E_{n}\right)(n=6,7,8), U_{q}\left(B_{3}\right) \subset U_{q}\left(F_{4}\right)$, it follows that there exist imaginary vectors for all types except $A_{1}, A_{2}, A_{3}, A_{4}, B_{2}$.

For types $A_{3}, B_{2}$ the basis $\mathbf{B}^{*}$ is explicitely described in [ $[\mathbf{B Z}]$ and $[\mathbf{R Z}, \overline{\mathbf{Z} 1}, \mathbf{C 1}]$ respectively, and it consists in all $q$-commuting products of elements of a certain finite subset $\mathcal{P}$ of $\mathbf{B}^{*}$. The elements of $\mathcal{P}$ may be regarded as the "prime vectors" of $\mathbf{B}^{*}$. A similar description also exists in type $A_{4}[\mathbf{Z 2}]$. From these descriptions one sees that all vectors of $\mathbf{B}^{*}$ are real if $\mathfrak{g}$ is of type $A_{n}(n \leqslant 4)$ or $B_{2}$.

In Section 3, we propose a conjecture regarding the multiplication by a real element of $\mathbf{B}^{*}$. This conjecture would imply that the Berenstein-Zelevinsky conjecture is true when one of the 
two factors is real. It would also yield an analogue of the Kashiwara crystal graph operator $\widetilde{e}_{i}$ for any real vector of $\mathbf{B}^{*}$.

Our original motivation for investigating the multiplicative properties of $\mathbf{B}^{*}$ came from some questions in the theory of finite dimensional representations of quantum affine algebras. Let $\widehat{\mathfrak{g}}$ be an affine Lie algebra and $U_{q}(\widehat{\mathfrak{g}})$ its quantum enveloping algebra. Let $V, W$ be finite dimensional irreducible representations of $U_{q}(\widehat{\mathfrak{g}})$. It is known that if $V \otimes W$ is irreducible then it is isomorphic to $W \otimes V$. Using our example of imaginary vector in type $A_{n}$, we are able to show that the converse is not true for $\widehat{\mathfrak{g}}=\widehat{\mathfrak{s l}}_{N}$, namely, we can exhibit an explicit irreducible representation $V$ such that $V \otimes V$ is not irreducible (see Section $\rrbracket$ ).

The name "imaginary vector" has been inspired by the recent seminal work of Fomin and Zelevinsky on cluster algebras [ [ZZ, Z3]. According to Zelevinsky [ [Z2], the general picture should be as follows. Let $G$ be the simply connected complex simple Lie group with Lie algebra $\mathfrak{g}$ and $N$ its maximal unipotent subgroup with Lie algebra $\mathfrak{n}$. The specialization $\mathbb{C}[N]$ of $U_{q}(\mathfrak{n})$ at $q=1$ (using the $\mathbb{Z}\left[q, q^{-1}\right]$-lattice $\mathcal{L}^{*}$ spanned by $\mathbf{B}^{*}$ ) is expected to be a cluster algebra, and to have finite cluster type only for $\mathfrak{g}$ of type $A_{n}(n \leqslant 4)$ or $B_{2}$. In this "finite case" the prime elements of $\mathbf{B}^{*}$ not belonging to the $q$-center are naturally labelled by the positive roots and the negatives of the simple roots of a certain complex simple Lie algebra $\mathfrak{c}$. In the "infinite case", it is expected that the imaginary prime vectors will correspond to imaginary roots of some infinite-dimensional Kac-Moody algebras.

\section{Examples of imaginary vectors}

2.1 Let $e_{i}(i=1, \ldots, r)$ be the Chevalley generators of $U_{q}(\mathfrak{n})$. Let $w_{0}$ denote the longest element of the Weyl group $W$ of $\mathfrak{g}$. To a reduced decomposition $w_{0}=s_{i_{1}} \cdots s_{i_{n}}$ is associated a convex ordering $\beta_{1}<\cdots<\beta_{n}$ of the positive roots of $\mathfrak{g}$ and a PBW-type basis of $U_{q}(\mathfrak{n})[\mathbf{L} \mathbf{1}]$ :

$$
E(\mathbf{m})=E\left(\beta_{1}\right)^{\left(m_{1}\right)} \cdots E\left(\beta_{n}\right)^{\left(m_{n}\right)}, \quad\left(\mathbf{m}=\left(m_{1}, \ldots, m_{n}\right) \in \mathbb{N}^{n}\right) .
$$

Let $\left\{E^{*}(\mathbf{m})\right\}$ be the dual PBW-basis, and let $\mathbf{b}(\mathbf{m})$ denote the unique element of $\mathbf{B}^{*}$ such that $\mathbf{b}(\mathbf{m})-E^{*}(\mathbf{m}) \in q L^{*}$, where $L^{*}$ is the $\mathbb{Z}[q]$-lattice spanned by $\mathbf{B}^{*}$.

We shall use repeatedly the following known fact (see $[\mathbf{R e}]$ ). For any $\mathbf{m}, \mathbf{p} \in \mathbb{N}^{n}$, the $\mathbf{B}^{*}$ expansion of $\mathbf{b}(\mathbf{m}) \mathbf{b}(\mathbf{p})$ contains the vector $\mathbf{b}(\mathbf{m}+\mathbf{p})$ with coefficient a power of $q$. Hence, if $\mathbf{b}(\mathbf{m}) \mathbf{b}(\mathbf{p}) \in q^{\mathbb{Z}} \mathbf{B}^{*}$ then $\mathbf{b}(\mathbf{m}) \mathbf{b}(\mathbf{p}) \cong \mathbf{b}(\mathbf{m}+\mathbf{p})$.

For $k \in \mathbb{N}^{*}$ and $b=\mathbf{b}(\mathbf{m}) \in \mathbf{B}^{*}$ we set $k \mathbf{m}=\left(k m_{1}, \ldots, k m_{n}\right)$ and $b^{[k]}=\mathbf{b}(k \mathbf{m})$. Thus $b$ is real if and only if $b^{2} \cong b^{[2]}$.

2.2 The following examples of imaginary vectors have been calculated with maple, using the algorithms described in [Le] for calculating the elements of $\mathbf{B}^{*}$ and their products. In [ [Le], the basis $\mathbf{B}^{*}$ is studied in the realization of $U_{q}(\mathfrak{n})$ in terms of quantum shuffles, following Rosso [Ro1, Ro2] and Green [G]. We will now explain very briefly the main features of this approach, refering the interested reader to $[\mathbf{L e}]$ for a detailed exposition.

In the quantum shuffle realization a vector $v \in U_{q}(\mathfrak{n})$ is represented by a $\mathbb{Q}(q)$-linear combination of words $w_{i_{1}} \ldots w_{i_{k}}$ over the alphabet $\left\{w_{1}, \ldots, w_{r}\right\}$. In particular, $e_{i}$ is identified with the 
letter $w_{i}$. In the sequel we shall write $w\left[i_{1}, \ldots, i_{k}\right]$ rather than $w_{i_{1}} \cdots w_{i_{k}}$. The multiplication of $U_{q}(\mathfrak{n})$ translates into the bilinear operation $*$ defined on words by

$$
w\left[i_{1}, \ldots, i_{m}\right] * w\left[i_{m+1}, \ldots, i_{m+n}\right]=\sum_{\sigma} q^{-e(\sigma)} w\left[i_{\sigma(1)}, \ldots, i_{\sigma(m+n)}\right]
$$

where the sum runs over the $\sigma \in \mathfrak{S}_{m+n}$ such that $\sigma(1)<\cdots<\sigma(m)$ and $\sigma(n+1)<\cdots<$ $\sigma(m+n)$, and

$$
e(\sigma)=\sum_{k \leqslant m<l ; \sigma(k)<\sigma(l)}\left(\alpha_{i_{\sigma(k)}}, \alpha_{i_{\sigma(l)}}\right) .
$$

Here, $\alpha_{1}, \ldots, \alpha_{r}$ are the simple roots of $\mathfrak{g}$ and $(\cdot, \cdot)$ denotes the bilinear form on the root lattice such that the entries of the Cartan matrix of $\mathfrak{g}$ are given by

$$
a_{i j}=\frac{2\left(\alpha_{i}, \alpha_{j}\right)}{\left(\alpha_{i}, \alpha_{i}\right)}=\frac{\left(\alpha_{i}, \alpha_{j}\right)}{d_{i}}, \quad(1 \leqslant i, j \leqslant r)
$$

where $d_{i}=\left(\alpha_{i}, \alpha_{i}\right) / 2 \in\{1,2,3\}$. When $q=1$, Equation (1) is nothing else than the classical shuffle product [ [Lo, Reu].

Following Rosso [ [Ro3] and Lalonde and Ram [[LR], it is shown in [Le] that to each total ordering of the set of simple roots corresponds a certain (dual) PBW basis, whose root vectors can be readily calculated using (11) by taking some iterated $q$-bracketings. (In the calculations below we take the natural order $\alpha_{1}<\cdots<\alpha_{r}$, which determines our choice of PBW basis.) Then, the characterization of $\mathbf{B}^{*}$ given in [ [e] (Proposition 41) yields an easy algorithm for calculating it. Moreover, in this setting, the products of elements of $\mathbf{B}^{*}$ and the calculation of their $\mathbf{B}^{*}$-expansion are rather simple to compute, thanks to the elementary formula (1) and to the fact that the words $w\left[i_{1}, \ldots, i_{k}\right]$ are linearly independent.

We will indicate below the main steps of the calculation of an imaginary vector of type $G_{2}$ in the quantum shuffle realization. In that case, the expressions of the vectors are so small that the computation could be done by hand. For the other types, the calculations are very similar but much bigger, so we shall omit them and give only the result.

2.3 type $G_{2}$ : The numbering of the simple roots $\alpha_{1}, \alpha_{2}$ is shown by the following Dynkin diagram:

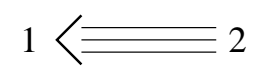

Thus, we have $\left(\alpha_{1}, \alpha_{1}\right)=2,\left(\alpha_{2}, \alpha_{2}\right)=6$. We choose the reduced decomposition

$$
w_{0}=s_{1} s_{2} s_{1} s_{2} s_{1} s_{2}
$$

which gives the following ordering of the positive roots:

$$
\alpha_{1}<3 \alpha_{1}+\alpha_{2}<2 \alpha_{1}+\alpha_{2}<3 \alpha_{1}+2 \alpha_{2}<\alpha_{1}+\alpha_{2}<\alpha_{2} .
$$


Let $b=\mathbf{b}(1,0,0,0,1,0)$. In the quantum shuffle realization, we have

$$
b=w[1,2,1],
$$

hence, using Equation (1) we obtain

$$
\begin{aligned}
b^{2}=2 & \left(1+q^{-2}\right) w[1,2,1,1,2,1]+\left(q^{4}+2 q^{2}+1+q^{-2}+2 q^{-4}+q^{-6}\right) w[1,1,2,2,1,1] \\
& +\left(q+2 q^{-1}+q^{-3}\right) w[1,2,1,2,1,1]+\left(q+2 q^{-1}+q^{-3}\right) w[1,1,2,1,2,1] .
\end{aligned}
$$

On the other hand, using the algorithm mentioned above for calculating $\mathbf{B}^{*}$, we get

$$
\begin{aligned}
b^{[2]}=(q & \left.+q^{-1}\right) w[1,2,1,1,2,1]+\left(q^{5}+2 q^{3}+q+q^{-1}+2 q^{-3}+q^{-5}\right) w[1,1,2,2,1,1] \\
& +\left(q^{2}+2+q^{-2}\right) w[1,2,1,2,1,1]+\left(q^{2}+2+q^{-2}\right) w[1,1,2,1,2,1] .
\end{aligned}
$$

Therefore, $b^{2} ¥ b^{[2]}$. More precisely, putting

$$
z=\left(q+q^{-1}\right) w[1,2,1,1,2,1]=\mathbf{b}(1,0,1,0,1,0),
$$

we obtain

$$
b^{2}=q^{-1}\left(b^{[2]}+z\right)
$$

hence $b$ is imaginary.

2.4 type $B_{3}$ : The numbering of the simple roots is given by the following Dynkin diagram:

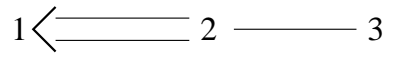

Thus, we have

$$
\left(\alpha_{1}, \alpha_{1}\right)=2,\left(\alpha_{2}, \alpha_{2}\right)=\left(\alpha_{3}, \alpha_{3}\right)=4 .
$$

We choose the reduced decomposition $w_{0}=s_{1} s_{2} s_{3} s_{1} s_{2} s_{1} s_{3} s_{2} s_{3}$, which gives the following ordering of the positive roots:

$\alpha_{1}<2 \alpha_{1}+\alpha_{2}<2 \alpha_{1}+\alpha_{2}+\alpha_{3}<\alpha_{1}+\alpha_{2}<2 \alpha_{1}+2 \alpha_{2}+\alpha_{3}<\alpha_{1}+\alpha_{2}+\alpha_{3}<\alpha_{2}<\alpha_{2}+\alpha_{3}<\alpha_{3}$.

Let $b=\mathbf{b}(0,1,0,0,0,0,0,1,0), z=\mathbf{b}(0,1,0,0,1,0,0,1,0)$. We have

$$
b^{2}=q^{-2}\left(b^{[2]}+z\right)
$$

hence $b$ is imaginary. 
2.5 type $C_{3}$ : The numbering of the simple roots is given by the following Dynkin diagram:

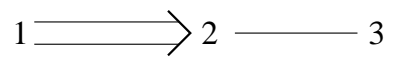

Thus, we have

$$
\left(\alpha_{1}, \alpha_{1}\right)=4,\left(\alpha_{2}, \alpha_{2}\right)=\left(\alpha_{3}, \alpha_{3}\right)=2 .
$$

We choose the reduced decomposition $w_{0}=s_{1} s_{2} s_{1} s_{3} s_{2} s_{1} s_{3} s_{2} s_{3}$, which gives the following ordering of the positive roots:

$\alpha_{1}<\alpha_{1}+\alpha_{2}<\alpha_{1}+2 \alpha_{2}<\alpha_{1}+\alpha_{2}+\alpha_{3}<\alpha_{1}+2 \alpha_{2}+\alpha_{3}<\alpha_{1}+2 \alpha_{2}+2 \alpha_{3}<\alpha_{2}<\alpha_{2}+\alpha_{3}<\alpha_{3}$.

Let $b=\mathbf{b}(0,1,0,0,0,0,0,1,0), z=\mathbf{b}(0,1,0,0,1,0,0,1,0)$. We have

$$
b^{2}=q^{-1}\left(b^{[2]}+z\right) \text {, }
$$

hence $b$ is imaginary.

2.6 type $D_{4}$ : The numbering of the simple roots is given by the following Dynkin diagram:

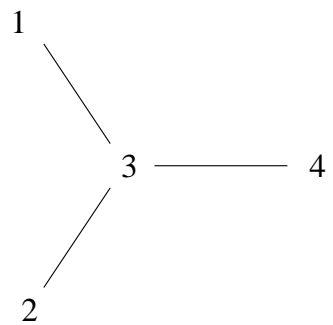

We choose the reduced decomposition $w_{0}=s_{1} s_{3} s_{2} s_{4} s_{3} s_{1} s_{4} s_{3} s_{2} s_{4} s_{3} s_{4}$, which gives the following ordering of the positive roots:

$$
\begin{aligned}
\alpha_{1}< & \alpha_{1}+\alpha_{3}<\alpha_{1}+\alpha_{2}+\alpha_{3}<\alpha_{1}+\alpha_{3}+\alpha_{4}<\alpha_{1}+\alpha_{2}+\alpha_{3}+\alpha_{4} \\
& <\alpha_{1}+\alpha_{2}+2 \alpha_{3}+\alpha_{4}<\alpha_{2}<\alpha_{2}+\alpha_{3}<\alpha_{2}+\alpha_{3}+\alpha_{4}<\alpha_{3}<\alpha_{3}+\alpha_{4}<\alpha_{4} .
\end{aligned}
$$

Let $b=\mathbf{b}(0,1,0,0,0,0,1,0,0,0,1,0), z=\mathbf{b}(0,1,0,0,1,0,0,1,0,0,1,0)$. We have

$$
b^{2}=q^{-1}\left(b^{[2]}+z\right)
$$

hence $b$ is imaginary.

2.7 type $A_{5}$ : The simple roots are denoted $\alpha_{1}, \alpha_{2}, \alpha_{3}, \alpha_{4}, \alpha_{5}$, and numbered in the natural way. We choose the reduced decomposition

$$
w_{0}=s_{1} s_{2} s_{3} s_{4} s_{5} s_{1} s_{2} s_{3} s_{4} s_{1} s_{2} s_{3} s_{1} s_{2} s_{1},
$$

which gives the following ordering of the positive roots:

$\alpha_{1}<\alpha_{1}+\alpha_{2}<\alpha_{1}+\alpha_{2}+\alpha_{3}<\alpha_{1}+\alpha_{2}+\alpha_{3}+\alpha_{4}<\alpha_{1}+\alpha_{2}+\alpha_{3}+\alpha_{4}+\alpha_{5}<\alpha_{2}<\alpha_{2}+\alpha_{3}<$ 


$$
\alpha_{2}+\alpha_{3}+\alpha_{4}<\alpha_{2}+\alpha_{3}+\alpha_{4}+\alpha_{5}<\alpha_{3}<\alpha_{3}+\alpha_{4}<\alpha_{3}+\alpha_{4}+\alpha_{5}<\alpha_{4}<\alpha_{4}+\alpha_{5}<\alpha_{5}
$$

Let

$$
\begin{aligned}
& b=\mathbf{b}(0,1,0,0,0,0,0,1,0,1,0,0,0,1,0) \\
& z=\mathbf{b}(0,1,0,1,0,0,1,0,1,0,1,0,0,1,0) .
\end{aligned}
$$

We have

$$
b^{2}=q^{-2}\left(b^{[2]}+z\right)
$$

hence $b$ is imaginary.

Remark 1 Let $\mathbf{B}$ be the canonical basis for $\mathfrak{g}$ of type $A_{5}$, and let $c$ and $t$ be the elements of $\mathbf{B}$ such that $\left(c, b^{[2]}\right)=(t, z)=1$. In [ [KS], Kashiwara and Saito prove that $t$ is a counterexample to a problem of Lusztig [L2], namely they show that the singular support $S S\left(L_{t}\right)$ of the irreducible perverse sheave $L_{t}$ corresponding to $t$ is not irreducible. More precisely, they show that

$$
S S\left(L_{t}\right)=\Lambda_{t} \cup \Lambda_{c}
$$

where $\Lambda_{t}$ and $\Lambda_{c}$ are the irreducible components of the nilpotent variety associated to $t$ and $c$. It would be interesting to understand the connection between Lusztig's problem and the conjecture of Berenstein and Zelevinsky.

Remark 2 In all the previous examples the vector $z \in \mathbf{B}^{*}$ occuring in the expansion of the imaginary vector $b$ is in fact an element of the $q$-center of $U_{q}(\mathfrak{n})$, that is, $z e_{i} \cong e_{i} z$ for every $i \in\{1, \ldots, r\}$ (see [C1] for a description of the $q$-center). This needs not to be true in general, since for example a $q$-central element in $U_{q}(\mathfrak{n})$ of type $A_{5}$ will no longer be $q$-central when we embed $U_{q}(\mathfrak{n})$ in $U_{q}\left(\mathfrak{n}^{\prime}\right)$ of type $A_{6}$.

\section{Real vectors and generalized crystal operators}

3.1 Let $b_{1}, b_{2}$ be elements of $\mathbf{B}^{*}$. Suppose that $b_{1}$ is real and $b_{1} b_{2} \notin q^{\mathbb{Z}} \mathbf{B}^{*}$.

Conjecture 1 The expansion of $b_{1} b_{2}$ on $\mathbf{B}^{*}$ is of the form

$$
b_{1} b_{2}=q^{m} b^{\prime}+q^{s} b^{\prime \prime}+\sum_{c \neq b^{\prime}, b^{\prime \prime}} \gamma_{b_{1} b_{2}}^{c}(q) c
$$

where $b^{\prime} \neq b^{\prime \prime}, m, s \in \mathbb{Z}, m<s, \gamma_{b_{1} b_{2}}^{c}(q) \in \mathbb{Z}\left[q, q^{-1}\right]$, and for all $c \in \mathbf{B}^{*}$ such that $\gamma_{b_{1} b_{2}}^{c}(q) \neq 0$

$$
\gamma_{b_{1} b_{2}}^{c}(q) \in q^{m+1} \mathbb{Z}[q] \cap q^{s-1} \mathbb{Z}\left[q^{-1}\right]
$$

It is known that the $\mathbf{B}^{*}$-expansion of $b_{2} b_{1}$ can be obtained from that of $b_{1} b_{2}$ by changing $q$ into $q^{-1}$ (and rescaling by an appropriate power of $q$ ) $[\mathbf{R e}]$. If $b_{1} b_{2} \notin q^{\mathbb{Z}} \mathbf{B}^{*}$ and Conjecture 1 is true, we see that $b_{2} b_{1}$ cannot be proportional to $b_{1} b_{2}$ since the term with the highest power of $q$ in $b_{1} b_{2}$ is $b^{\prime \prime}$, while that in $b_{2} b_{1}$ is $b^{\prime} \neq b^{\prime \prime}$. Hence Conjecture 1 w would imply that the Berenstein-Zelevinsky conjecture holds true when one of the two vectors is real. It is proved in [LNT] that if $\mathfrak{g}$ is of type $A_{n}$, the quantum flag minors are real elements of $\mathbf{B}^{*}$. Hence our conjecture agrees with the recent result of Caldero [C2] showing that if a quantum flag minor $q$-commutes with an arbitrary element of $\mathbf{B}^{*}$, then their product belongs to $q^{\mathbb{Z}} \mathbf{B}^{*}$. 


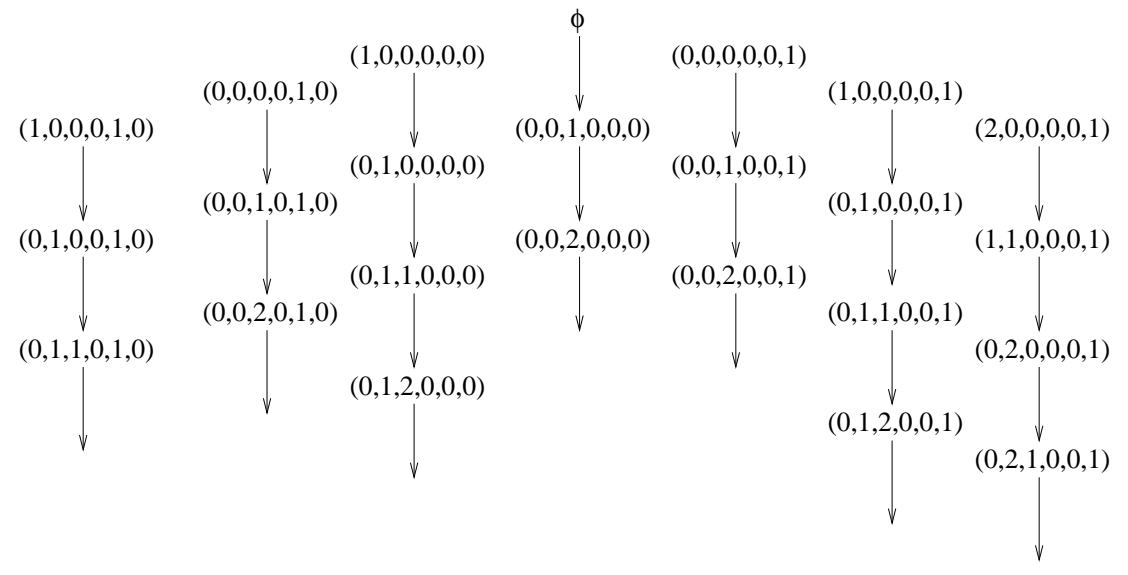

Figure 1: The first $b_{1}$-strings with $b_{1}=\mathbf{b}(0,0,1,0,0,0)$ in type $G_{2}$.

3.2 Using results of Kashiwara ([ [K2], $5.3 .1)$, it is easy to check Conjecture 1 in the case where $b_{1}=e_{i}$ is a Chevalley generator of $U_{q}(\mathfrak{n})$ (note that $e_{i}$ is always a real element of $\mathbf{B}^{*}$ ). In that case, $b^{\prime}=\widetilde{e}_{i}\left(b_{2}\right)$, where $\widetilde{e}_{i}$ is the Kashiwara operator associated with the simple root vector $e_{i}$. (Here we are abusing notation by denoting in the same way a vector of $\mathbf{B}^{*}$ and the corresponding element of the crystal basis at $q=0$.) Similarly $b^{\prime \prime}=\widetilde{e}_{i}^{\tau}\left(b_{2}\right)$, where $\widetilde{e}_{i}^{\tau}=\tau \widetilde{e}_{i} \tau$ and $\tau$ is the involution of $\mathbf{B}^{*}$ induced by the anti-automorphism of $U_{q}(\mathfrak{n})$ which fixes the $e_{i}$ 's.

Assuming Conjecture 11, let us write $b^{\prime}=b_{1} \diamond b_{2}$ and $b^{\prime \prime}=b_{2} \diamond b_{1}$. (If $b_{1} b_{2} \cong c \in \mathbf{B}^{*}$, we put $b_{1} \diamond b_{2}=b_{2} \diamond b_{1}=c$.) For a fixed real $b_{1}$, we may regard the maps $b \mapsto b_{1} \diamond b$ and $b \mapsto b \diamond b_{1}$ as generalized left and right Kashiwara operators associated to $b_{1}$. (Note that for every positive root $\beta$, the vector $E^{*}(\beta)$ of the dual PBW-basis is a real vector in $\mathbf{B}^{*}$. Thus, in particular, we obtain conjectural analogues of Kashiwara operators for all positive roots).

Conjecture 2 The maps $b \mapsto b_{1} \diamond b$ and $b \mapsto b \diamond b_{1}$ are injective.

Conjecture 2 implies that we can partition the set $\mathbf{B}^{*}$ into infinite $b_{1}$-strings:

$$
b \longrightarrow b_{1} \diamond b \longrightarrow b_{1} \diamond\left(b_{1} \diamond b\right) \longrightarrow \cdots
$$

with $b$ running over $\mathbf{B}^{*}-\left(b_{1} \diamond \mathbf{B}^{*}\right)$. This is illustrated in Figure 1 for $b_{1}=\mathbf{b}(0,0,1,0,0,0)$ in type $G_{2}$. We also have a similar string decomposition corresponding to the right operation $b \mapsto b \diamond b_{1}$.

3.3 These two conjectures are based on extensive computer calculations.

In particular, Conjecture 1 has been checked systematically in type $G_{2}$ for all vectors $b_{1}, b_{2}$ of principal degree $\leqslant 7$ which are not both imaginary. Note that among the 116 vectors of $\mathbf{B}^{*}$ of degree $\leqslant 7$, there are 10 imaginary vectors:

$$
\mathbf{b}(1,0,0,0,1, j), \quad(0 \leqslant j \leqslant 4),
$$




$$
\begin{array}{ll}
\mathbf{b}(2,0,0,0,2, j), & (0 \leqslant j \leqslant 1), \\
\mathbf{b}(j, 1,0,0,0,1), & (0 \leqslant j \leqslant 2) .
\end{array}
$$

Only 3 of them are prime, namely $\mathbf{b}(1,0,0,0,1,0), \mathbf{b}(2,0,0,0,2,0)$ and $\mathbf{b}(0,1,0,0,0,1)$.

\section{Applications to affine Hecke algebras and quantum affine algebras}

One important motivation for studying the multiplicative properties of $\mathbf{B}^{*}$ comes from the representation theory of affine Hecke algebras and quantum affine algebras [LNT].

4.1 Let $H_{m}(t)$ be the affine Hecke algebra of type $G L_{m}$ over $\mathbb{C}$, where $t \in \mathbb{C}^{*}$ is of infinite multiplicative order. It has invertible generators $T_{1}, \ldots, T_{m-1}, y_{1}, \ldots, y_{m}$ subject to the relations

$$
\begin{array}{ll}
T_{i} T_{i+1} T_{i}=T_{i+1} T_{i} T_{i+1}, & (1 \leqslant i \leqslant m-2), \\
T_{i} T_{j}=T_{j} T_{i}, & (|i-j|>1), \\
\left(T_{i}-t\right)\left(T_{i}+1\right)=0, & (1 \leqslant i \leqslant m-1), \\
y_{i} y_{j}=y_{j} y_{i}, & (1 \leqslant i, j \leqslant m), \\
y_{j} T_{i}=T_{i} y_{j}, & (j \neq i, i+1), \\
T_{i} y_{i} T_{i}=t y_{i+1}, & (1 \leqslant i \leqslant m-1) .
\end{array}
$$

Let $\mathcal{C}_{m}$ denote the category of finite-dimensional $H_{m}(t)$-modules, and $\mathcal{C}_{m, r}$ the sub-category consisting of those modules for which all eigenvalues of the pairwise commutative generators $y_{1}, \ldots, y_{m}$ belong to $\left\{t, t^{2}, \ldots, t^{r}\right\}$. Let $\mathcal{R}_{r}$ be the direct sum of the complexified Grothendieck groups of the $\mathcal{C}_{m, r}(m \in \mathbb{N})$, endowed with the multiplication given by the induction functors $\mathcal{C}_{m, r} \times \mathcal{C}_{n, r} \longrightarrow \mathcal{C}_{m+n, r}$. The algebra $\mathcal{R}_{r}$ is graded by deciding that the classes of the $H_{m}(t)-$ modules have degree $m$.

Let $N$ be the group of upper triangular unipotent $(r+1) \times(r+1)$-matrices, $\mathfrak{n}$ the Lie algebra of $N$, and $U_{q}(\mathfrak{n})$ the corresponding quantum enveloping algebra. (This is the positive part of a quantum enveloping algebra of type $A_{r}$.) Let $\mathcal{L}^{*}$ be the $\mathbb{Z}\left[q, q^{-1}\right]$-lattice spanned by the dual canonical basis $\mathbf{B}^{*}$ of $U_{q}(\mathfrak{n})$. It is well-known that the specialization $\mathbb{C} \otimes_{\mathbb{Z}\left[q, q^{-1}\right]} \mathcal{L}^{*}$, where $\mathbb{C}$ is regarded as a $\mathbb{Z}\left[q, q^{-1}\right]$-module via $q \mapsto 1$, is isomorphic to the algebra $\mathbb{C}[N]$ of regular functions on the group $N$. Let $\mathcal{B}^{*} \subset \mathbb{C}[N]$ denote the specialization at $q=1$ of $\mathbf{B}^{*}$ obtained in this way.

It was essentially shown by Zelevinsky [ [Z4] that $\mathcal{R}_{r}$ and $\mathbb{C}[N]$ are isomorphic as graded algebras. Moreover, by a dual version of Ariki's theorem [ $\mathbf{A}, \overline{\mathbf{L N T}}]$, the basis of $\mathcal{R}_{r}$ given by the classes of the simple modules is mapped to $\mathcal{B}^{*}$ under this isomorphism. Therefore, for $\mathfrak{g}$ of type $A_{r}$, the multiplication of two elements of respective principal degree $m$ and $n$ of $\mathbf{B}^{*}$ encodes the induction product of two simple objects of the categories $\mathcal{C}_{m, r}$ and $\mathcal{C}_{n, r}$.

4.2 Consider the example of 2.7 for type $A_{5}$. The vector $b$ has principal degree 8 . Therefore, under the above isomorphism it corresponds to a simple object $M$ of the category $\mathcal{C}_{8,5}$. The fact that $b$ is imaginary translates into the fact that the induction square of $M$ is not simple. In 
the Zelevinsky parametrization of simple modules by multi-segments, the $H_{8}(t)$-module $M$ is labelled by the multi-segment

$$
[1,2],[2,3,4],[3],[4,5] \text {. }
$$

It has dimension 252. Its induction square $M \odot M$ is a $H_{16}(t)$-module with two composition factors: the 2522 520-dimensional simple module $M^{\prime}$ labelled by

$$
[1,2],[2,3],[3,4],[4,5],[1,2,3,4],[2,3,4,5]
$$

and the 814773960 -dimensional simple module $M^{\prime \prime}$ labelled by

$$
[1,2],[1,2],[2,3,4],[2,3,4],[3],[3],[4,5],[4,5] \text {. }
$$

4.3 Let $U_{q}\left(\widehat{\mathfrak{s l}}_{N}\right)$ be the quantized affine algebra of type $A_{N-1}^{(1)}$ with parameter $q$ a square root of $t$ (see for example $[\mathbf{C P}]$ for the defining relations of $U_{q}\left(\widehat{\mathfrak{s l}}_{N}\right)$ ). The quantum affine Schur-Weyl duality between $H_{m}(t)$ and $U_{q}\left(\widehat{\mathfrak{s l}}_{N}\right)[\mathbf{C P}, \mathbf{C h}, \mathbf{G R V}]$ gives a functor $\mathcal{F}_{m, N}$ from the category $\mathcal{C}_{m}$ to the category of level 0 finite-dimensional representations of $U_{q}\left(\widehat{\mathfrak{s l}}_{N}\right)$. If $N \geqslant m, \mathcal{F}_{m, N}$ maps the non-zero simple modules of $\widehat{H}_{m}(t)$ to non-zero simple modules of $U_{q}\left(\widehat{\mathfrak{s l}}_{N}\right)$. Moreover, for $M_{1}$ in $\mathcal{C}_{m_{1}}$ and $M_{2}$ in $\mathcal{C}_{m_{2}}$ one has

$$
\mathcal{F}_{m_{1}+m_{2}, N}\left(M_{1} \odot M_{2}\right)=\mathcal{F}_{m_{1}, N}\left(M_{1}\right) \otimes \mathcal{F}_{m_{2}, N}\left(M_{2}\right) .
$$

Let $N \geqslant 16$. The image of the $H_{8}(t)$-module $M$ under $\mathcal{F}_{8, N}$ is a finite-dimensional irreducible representation $V$ of $U_{q}\left(\widehat{\mathfrak{s l}}_{N}\right)$ such that $V \otimes V$ is not irreducible. The Drinfeld polynomials parametrizing $V$ in the classification of Chari and Pressley are

$$
\begin{aligned}
& P_{1}(u)=u-q^{-6}, \\
& P_{2}(u)=\left(u-q^{-3}\right)\left(u-q^{-9}\right), \\
& P_{3}(u)=u-q^{-6} \\
& P_{k}(u)=1, \quad(k \geqslant 4) .
\end{aligned}
$$

The composition factors of $V \otimes V$ are the images $V^{\prime}$ and $V^{\prime \prime}$ of $M^{\prime}$ and $M^{\prime \prime}$ under $\mathcal{F}_{16, N}$. The Drinfeld polynomials of $V^{\prime}$ are

$$
\begin{aligned}
& P_{2}(u)=\left(u-q^{-3}\right)\left(u-q^{-5}\right)\left(u-q^{-7}\right)\left(u-q^{-9}\right) \\
& P_{4}(u)=\left(u-q^{-5}\right)\left(u-q^{-7}\right) \\
& P_{k}(u)=1, \quad(k \neq 2,4)
\end{aligned}
$$

and those of $V^{\prime \prime}$ are

$$
\begin{aligned}
& P_{1}(u)=\left(u-q^{-6}\right)^{2}, \\
& P_{2}(u)=\left(u-q^{-3}\right)^{2}\left(u-q^{-9}\right)^{2}, \\
& P_{3}(u)=\left(u-q^{-6}\right)^{2}, \\
& P_{k}(u)=1, \quad(k \geqslant 4) .
\end{aligned}
$$


In fact, since the highest non trivial Drinfeld polynomial of $V^{\prime \prime}$ is $P_{4}(u)$, we see that $V \otimes V$ is not simple for any $N \geqslant 5$. For $N=5$, we have

$$
\operatorname{dim} V=1995, \quad \operatorname{dim} V^{\prime}=7350, \quad \operatorname{dim} V^{\prime \prime}=3972675 .
$$

4.4 The meaning of Conjecture 1 in this setting should be as follows.

Conjecture 3 Let $V$ and $W$ be finite-dimensional irreducible representations of $U_{q}\left(\widehat{\mathfrak{s l}}_{N}\right)$. Suppose that $V \otimes V$ is irreducible. Then $V \otimes W$ has simple socle $S$ and simple head H. Moreover if $V \otimes W$ is not simple $S$ and $H$ are not isomorphic.

\section{Acknowledgements}

I thank M. Kashiwara and A. Zelevinsky for fruitful discussions. In particular, I am grateful to A. Zelevinsky who convinced me, after I found an example of imaginary vector in type $G_{2}$, that imaginary vectors should also exist for other types.

This note was written during my visit to M.S.R.I. Berkeley to participate in the program "Infinite dimensional algebras and mathematical physics", organized by E. Frenkel, V. Kac, I. Penkov, V. Serganova and G. Zuckerman. I thank the organizers for inviting me.

\section{References}

[A] S. ARIKI, On the decomposition numbers of the Hecke algebra of $G(n, 1, m)$, J. Math. Kyoto Univ. 36 (1996), $789-808$.

[BZ] A. Berenstein, A. Zelevinsky, String bases for quantum groups of type $A_{r}$, Adv. Soviet Mathematics, 16 (1993), $51-89$.

[C1] P. CALDERO, Adapted algebras for the Berenstein-Zelevinsky conjecture, Preprint 2001, math.RT/0104165.

[C2] P. CALDERO, A multiplicative property of quantum flag minors, Preprint 2001, math.RT/0112205.

[CP] V. Chari, A. Pressley, Quantum affine algebras and affine Hecke algebras, Pacific J. Math. 174 (1996), 295-326.

[Ch] I. V. Cherednik, A new interpretation of Gelfand-Tzetlin bases, Duke Math. J., 54 (1987), 563-577.

[FZ] S. Fomin, A. Zelevinsky, Cluster algebras I: Foundations, to appear in J. Amer. Math. Soc., math.RT/0104151.

[GRV] V. GinZburg, N. Yu Reshetikhin, E. Vasserot, Quantum groups and flag varieties, A.M.S. Contemp. Math. 175 (1994), 101-130.

[G] J. A. Green, Quantum groups, Hall algebras and quantum shuffles, in 'Finite reductive groups' (Luminy 1994), 273-290, Birkhäuser Prog. Math. 141, 1997.

[K1] M. KaShiWARA, On crystal bases of the q-analogue of universal enveloping algebras, Duke Math. J. 63 (1991), 465-516.

[K2] M. KaShiwara, Global bases of quantum groups, Duke Math. J. 69 (1993), 455-485.

[K3] M. KASHIWARA, On level zero representations of quantized affine algebras, (2000), math.QA/0010293.

[KS] M. Kashiwara, Y. SAIto, Geometric construction of crystal bases, Duke Math. J. 89 (1997), 9-36. 
[LR] P. Lalonde, A. Ram, Standard Lyndon bases of Lie algebras and enveloping algebras, Trans. Amer. Math. Soc. 347 (1995), 1821-1830.

[Le] B. LECLERC, Dual canonical bases, quantum shuffles and q-characters, Preprint 2002.

[LNT] B. LeCLERC, M. NAZAROV, J.-Y. ThiBon, Induced representations of affine Hecke algebras and canonical bases of quantum groups, to appear in 'Studies in memory of Issai Schur', Birkhäuser 2002.

[Lo] M. Lothaire, Combinatorics on words, Readings, Massachusetts, 1983.

[L1] G. LUSZTIG, Introduction to quantum groups, Birkhäuser 1993.

[L2] G. LusZTig, Quivers, perverse sheaves and quantized enveloping algebras, J. Amer. Math. Soc. 4, (1991), 365-421.

[Re] M. REINEKE, Multiplicative properties of dual canonical bases of quantum groups, J. Algebra 211 (1999), 134-149.

[RZ] V. RETAKH, A. ZELEVINSKy, The base affine space and canonical bases in irreducible representations of the group $S p(4)$, Soviet Math. Dokl. 37 (1988), 618-622.

[Reu] C. Reutenauer, Free Lie algebras, Oxford University Press, 1993.

[Ro1] M. Rosso, Groupes quantiques et algèbres de battage quantiques, C. R. Acad. Sci. Paris 320 (1995), 145-148.

[Ro2] M. Rosso, Quantum groups and quantum shuffles, Invent. Math. 133 (1998), 399-416.

[Ro3] M. Rosso, Lyndon bases and the multiplicative formula for R-matrices, Preprint 2002.

[Z1] A. Zelevinsky, Connected components of real double Bruhat cells, Intern. Math. Res. Notices, 21 2000, 1131-1153.

[Z2] A. Zelevinsky, Personal communication.

[Z3] A. ZELEVINSKy, From Littlewood-Richardson coefficients to cluster algebras in three lectures, math.RT/0112062.

[Z4] A. ZELEVINSKY, Induced representations of reductive p-adic groups II, Ann. Sci. E.N.S. 13 (1980), 165-210.

B. LeCLeRC : Département de Mathématiques, Université de Caen, Campus II, Bld Maréchal Juin, BP 5186, 14032 Caen cedex, France

email : leclerc@math.unicaen.fr 